\title{
Self-condensation culture for vascularized organoid
}

\author{
Shoichiro Sumi \\ Institute for Frontier Life and Medical Sciences, Kyoto University, Kyoto, Japan \\ Correspondence to: Shoichiro Sumi, MD, PhD. Institute for Frontier Life and Medical Sciences, Kyoto University, 53 Shogoin-Kawahara-cho, Sakyo- \\ ku, Kyoto 606-8507, Japan. Email: sumi@infront.kyoto-u.ac.jp. \\ Provenance: This is an invited Editorial commissioned by Section Editor Liuhua Zhou (Department of Urology, Nanjing First Hospital, Nanjing \\ Medical University, Nanjing, China). \\ Comment on: Takahashi Y, Sekine K, Kin T, et al. Self-Condensation Culture Enables Vascularization of Tissue Fragments for Efficient Therapeutic \\ Transplantation. Cell Rep 2018;23:1620-9.
}

Submitted Aug 22, 2018. Accepted for publication Sep 07, 2018.

doi: 10.21037/atm.2018.09.12

View this article at: http://dx.doi.org/10.21037/atm.2018.09.12

Islet transplantation is an essential treatment for type 1 diabetes. However, low engraftment of transplanted islets makes it difficult to maximize the treatment efficacy. Prof. Taniguchi's research group has developed a method to make tissue organoids including pancreatic islets in vitro, using mesenchymal stem cells (MSCs) and human umbilical cordderived endothelial cells (HUVECs) (1). They successfully generated complex tissues including not only islets but also various organ fragments such as liver, kidney, heart, brain, and others. The self-condensation culture of islets with MSCs and HUVECs promoted islet function in vitro and massively improved engraftment after transplantation through rapid induction of functional vascular networks. This in vitro method to fabricate vascularized organoids offers us a promising means of therapeutic tissue transplantation, e.g., pancreatic islets transplantation to treat diabetes.

This approach was first reported by Takebe et al. to fabricate liver buds (2). After that, the similar approach was further applied to the formation of vascularized islet tissues. In addition to the improvement of graft survival, the vascularized islet tissues also restored fulminant diabetic mice to euglycemia. Furthermore, the diabetic animals received the vascularized islet tissues had a higher insulin secretion, a better glucose tolerance, and a fast blood glucose disappearance rate when compared to those transplanted with non-vascularized (simply isolated) islets. The vascularized islet tissues induced functional vascular networks earlier in vivo, and this shall be the key for successful engraftment. Furthermore, although the details await further investigation, this approach can also create organoids from other tissue sources such as brain, heart, intestine, kidney, and lung, in addition to islets and liver. This approach extends the therapeutic applications of vascularized tissues to various diseases whose curative treatments have not been established.

In order to increase engraftment of transplanted islets, co-transplantation of MSCs (3), use of growth factors (4) and pre-vascularized transplantation site $(5,6)$ have been reported. However, Prof. Takebe's approach seem to include most of these advantages, since MSCs co-cultured with islets secrete growth factors to protect islets and already formed vascularization in the graft induces early vascular network formation like a pre-vascularized transplantation site. Moreover, self-condensed tissue may provide pretty physiological cellular environment through cell-cell and cell-ECM relationship just like native islets in situ before transplantation.

Although this approach seems promising to increase engraftment of normally vascularized tissues, it cannot be applied to non-vascularized tissues, e.g., cartilage and nucleus pulposus. In such cases, tissue engineered scaffolds play important roles. It is well-known that the transplanted cells are difficult to be anchored at the injury site, if single cells are delivered. For example, chondrocyte injection for the treatment of cartilage defect generally results in poor outcomes while a cell carrier, such as the tissue engineered scaffold, can improve the cell adhesion as well as histomorphometric findings (7). On the other hand, the degradation of tissue engineered scaffold may influence the 
surrounding cellular environment and subsequently cause proinflammatory reaction, which can impair the integration of transplanted tissues to the transplant site. Therefore, this approach has an advantage because of the scaffold-free formation of a $3 \mathrm{D}$ tissue construct (organoid). The MSCdependent contraction forming the self-organized tissues may benefit cell/tissue replacement therapy in other various conditions.

This approach used two other cell types (MSCs and HUVECs) in addition to the primary functioning cells, e.g., islets and hepatocytes. Therefore, when considering clinical application, sources of MSCs and HUVECs may raise another issue. MSCs can be obtained from various tissues including bone marrow and adipose tissue. On the other hand, HUVECs are obtained from human umbilical cord. Therefore, the donors of these cells are ordinarily different, unless MSCs are obtained from the appendage of the same fetus as HUVECs while the islets shall still come from another donor. So, sensitization to alloantigens may make immunosuppression more difficult.

Lineage-directed inductions from undifferentiated [embryonic stem (ES) or induced pluripotent stem (iPS)] cells to MSCs (8) and endothelial cells (9) have been studied. If these two cell types and functional cell, e.g., islets, are differentiated from the same undifferentiated cell line, this organoid can be made from a single donor source. This may be the ultimate goal of the organoid formation. In fact, they recently made liver buds from iPS cells and investigated its feasibility as a hepatitis B virus (HBV) infection model (10). In Japan, HLA-homo iPS cell bank is being established for common HLA types in Japanese. Then, HLA-matched patients can be treated with organoids made from these HLA-homo iPS cells. However, this kind of treatment will be extremely expensive and it is uncertain if the society can afford such costs for a number of patients, even though the cost of individual iPS cell induction from each patient can be saved by HLA-homo iPS cell bank. Expense issues of this kind of regenerative medicine await right answer, while several clinical trials using various iPS-derived cell types are recently proposed in addition to retinal pigment epithelium.

Spontaneously organized tissue including islets through self-condensation showed much enhanced in vitro function in comparison to simple isolated islets (1). This means that their organoids have better function than simple cell spheroids without blood circulation. The mechanisms of this functional improvement seem interesting and wonderful target of future investigations. On the other hand, islets can be micro- or macro-encapsulated and such encapsulated islets are expected to cure diabetes without immunosuppression (11). Accordingly, the organoid including islets may be suitable for encapsulated islets. However, in such situation, without blood circulation, supply of oxygen and nutrients, and clearance of waste products depend on simple diffusion. Therefore, their organoids are not suitable for simple encapsulation, since islet-supporting MSCs and HUVECs compete oxygen and nutrients to survive and the size of their organoids (about $1 \mathrm{~mm}$ in diameter) seem too large to avoid central necrosis (12). On the other hand, such organoids may be suitable for organoid on a chip (13) to be used for pharmacological and toxicological purposes, if proper oxygen supply is maintained. Their recent publication of liver buds as a HBV infection model (10) may be highly estimated in this context.

In conclusion, recent publication by Prof. Taniguchi's research group (1) showed organoid formation through self-condensation and self-organization induced by MSCs and HUVECs. Organoids including islets showed early engraftment and high function to cure diabetic animals after transplantation. Such organoids can include not islets but other tissues like liver, kidney, lung, and so on. This method seems a breakthrough toward new treatments using tissue transplantation and new drug screening methods using organoid on a chip.

\section{Acknowledgements}

I thank Prof. Yang KC for his valuable comments and discussion.

\section{Footnote}

Conflicts of Interest: The author has no conflicts of interest to declare.

\section{References}

1. Takahashi Y, Sekine K, Kin T, et al. Self-condensation culture enables vascularization of tissue fragments for efficient therapeutic transplantation. Cell Rep 2018;23:1620-9.

2. Takebe T, Zhang RR, Koike H, et al. Generation of a vascularized and functional human liver from an iPSCderived organ bud transplant. Nat Protoc 2014;9:396-409.

3. Berman DM, Willman MA, Han D, et al. Mesenchymal stem cells enhance allogeneic islet engraftment in 
nonhuman primates. Diabetes 2010;59:2558-68.

4. Fiaschi-Taesch NM, Berman DM, Sicari BM, et al. Hepatocyte growth factor enhances engraftment and function of nonhuman primate islets. Diabetes 2008;57:2745-54.

5. Kawakami Y, Iwata H, Gu YJ, et al. Modified subcutaneous tissue with neovascularization is useful as the site for pancreatic islet transplantation. Cell Transplant 2000;9:729-32.

6. Pepper AR, Gala-Lopez B, Pawlick R, et al. A prevascularized subcutaneous device-less site for islet and cellular transplantation. Nat Biotechnol 2015;33:518-23.

7. Wang CC, Yang KC, Lin KH, et al. Expandable Scaffold Improves Integration of Tissue-Engineered Cartilage: An In Vivo Study in a Rabbit Model. Tissue Eng Part A 2016;22:873-84.

8. Li O, Tormin A, Sundberg B, et al. Human embryonic stem cell-derived mesenchymal stroma cells (hES-

MSCs) engraft in vivo and support hematopoiesis without suppressing immune function: implications for off-the

Cite this article as: Sumi S. Self-condensation culture for vascularized organoid. Ann Transl Med 2018;6(Suppl 1):S15. doi: 10.21037/atm.2018.09.12 shelf ES-MSC therapies. PLoS One 2013;8:e55319.

9. Narazaki G, Uosaki H, Teranishi M, et al. Directed and systematic differentiation of cardiovascular cells from mouse induced pluripotent stem cells. Circulation 2008;118:498-506.

10. Nie YZ, Zheng YW, Miyakawa K, et al. Recapitulation of hepatitis $\mathrm{B}$ virus-host interactions in liver organoids from human induced pluripotent stem cells. EBioMedicine 2018. [Epub ahead of print].

11. Yang KC, Yanai G, Yang SY, et al. Low-adhesive ethylene vinyl alcohol-based packaging to xenogeneic islet encapsulation for type 1 diabetes treatment. Biotechnol Bioeng 2018;115:2341-55.

12. Iwata H, Arima Y, Tsutsui Y. Design of Bioartificial Pancreases From the Standpoint of Oxygen Supply. Artif Organs 2018;42:E168-85.

13. Skardal A, Shupe T, Atala A. Organoid-on-a-chip and body-on-a-chip systems for drug screening and disease modeling. Drug Discov Today 2016;21:1399-411. 